\title{
螺旋内齿圈拉刀精切齿磨削方法研究
}

\author{
贾 康 ${ }^{1,2}$ 张银行 ${ }^{2}$ 南凯刚 ${ }^{3}$ 洪 军 1,2 \\ (1. 西安交通大学现代设计及转子轴承系统教育部重点实验室 西安 710049 ;
}

2. 西安交通大学机械工程学院 西安 710049;

3. 西安交通大学科技与教育发展研究院 西安 710049)

\begin{abstract}
摘要: 螺旋内齿圈拉刀是加工螺旋内齿圈的重要工具, 其精切齿的齿升量结构复杂、齿形精度要求高, 对磨削制造的效率和 质量提出挑战。针对螺旋内齿圈拉刀的精切齿磨削加工，在对切削齿进行几何结构与刀体分布建模的基础上，以保证磨削表 面质量、提升加工效率、易于程序化等为目标, 剖析磨削工艺与保障策略, 设计出适用于拉刀精切齿精细结构的无干涉磨削 工艺与路径实现方法。基于螺旋内齿圈拉刀精切段的双螺旋结构进行切削齿坐标的参数化建模, 并对精切齿非对称齿升量的 分布模型进行解析与建模。从制造工艺保障与加工效率提升等角度对精切齿的磨削工序进行分析与规划, 并设计优化的磨削 加工路径, 同时提出参数化驱动的精切齿磨削路径控制算法。此外, 基于成型磨削接触线对精切齿侧面完备且无干涉磨削的 行程进行分析与计算, 并进一步针对可行砂轮半径最大化进行磨削工艺条件的优化分析。最后, 通过计算一把螺旋拉刀的磨 削参数与磨削工艺条件对本研究的实用性进行验证。
\end{abstract}

关键词: 螺旋内齿圈; 拉刀; 磨削工艺; 路径规划; 干涉回避; 砂轮优选

中图分类号: TH132

\section{Research on Grinding Method of Finishing Cutting Teeth for Internal Helical Ring Gear Broach Tools}

\author{
JIA Kang $^{1,2}$ ZHANG Yinhang ${ }^{2}$ NAN Kaigang ${ }^{3}$ HONG Jun ${ }^{1,2}$ \\ (1. Key Laboratory of Education Ministry for Modern Design \& Rotor-bearing System, \\ Xi'an Jiaotong University, Xi'an 710049; \\ 2. School of Mechanical Engineering, Xi'an Jiaotong University, Xi'an 710049; \\ 3. Research and Education Development Institute, Xi'an Jiaotong University, Xi'an 710049)
}

\begin{abstract}
Internal helical ring gear broach tool is a crucial tool for helical ring gear manufacturing. The complex rise-per-tooth pattern and the high precise of finishing cutting teeth result produce challenge to machining efficiency and quality of surface. In the basis of modelling for the teeth geometry and distribution, this paper planned the interference-free grinding process and path for finishing cutting teeth of broaching tool with investigating the grinding process and ensuring strategy which are aim to ensure the quality of surface, improve the machining efficiency, and easy to programming. Firstly, the mathematic modelling for teeth coordinate is performed based on the dual-spiral distribution of broach tool, and the asymmetric step pattern of rise-per-tooth of finishing cutting teeth is established. Then, the grinding process is analyzed and the optimal grind path is planned with respect the maintain of process and machining efficiency, meanwhile, the parametric-driven algorithm for finishing cutting teeth grinding is developed. Besides, with combing the instant contact line of grinding wheel, the interference-free grinding travel that ensures the complete tooth surfaces machining is calculated, and the optimal condition for maximizing the radius of grinding wheel is investigated in further. At last, the grinding parameters and the optimization conditions are identified for a helical broach tool to verify the effectiveness of this method.
\end{abstract}

Key words: internal helical ring gear; broaching tool; grinding process; path planning; interference avoidance; wheel optimization

\footnotetext{
* 国家自然科学基金 (51705403)、中央高校基本科研业务费专项资金 (xjh012019003) 和中国博士后科学基金(2017M623158) 资助项目。 20190619 收到初稿, 20191209 收到修改稿
} 


\section{1 前言}

螺旋内齿圈是轿车自动变速器中行星轮系的重 要组成零件, 其加工精度直接决定了变速器的工作 性能。现今螺旋内齿圈的加工主要采用插齿 ${ }^{[1]}$ 、车 齿 ${ }^{[2-3]}$ 、螺旋拉削 ${ }^{[4]}$ 和锻造 ${ }^{[5]}$ 等方法。插齿加工效率 相对较低, 滚齿缺乏加工内齿圈能力, 车齿当前研 究与应用相对较少, 而为保证齿轮精度冷锻工艺复 杂且成本较高, 相较而言, 螺旋拉削加工效率极高 且能够保证稳定的 7 8 级加工精度, 在大批量制造 模式具有良好的经济性。

螺旋内齿圈拉刀以螺旋内齿圈的齿形一次性拉 削成型为目标, 融多种齿形的粗切与渐开线廓形的 精切成型为一体, 将拉削工艺发挥到了极高的水平。 其切削齿结构数量巨大, 切削齿齿形结构与分布模 式复杂, 尤以精切齿形状精度要求极高。由拉削加 工原理可知, 精切齿的良好制造直接决定着螺旋内 齿圈的加工品质。因此, 螺旋内齿圈拉刀精切齿的 精密制造, 及其高效率实现是螺旋内齿圈拉刀制造 技术的关键。当前，全球仅有德国的 Arthur Klink、 日本的 Mitsubishi 和 NACHI 以及美国的 Federal Broach 等为数家厂家具备螺旋内齿圈拉刀的制造能 力, 然而其昂贵的造价与较长的制造周期以及配套 的刃磨服务缺失等对我国变速器研发等产生一定制 约。由此可见, 研究螺旋内齿圈拉刀的高精度高效 磨削加工方法至关重要。GONÇALVES 等 ${ }^{[6]}$ 从拉削 力建模角度对于简单的螺旋内齿圈拉刀结构进行了 基本阐述, 表明刀具的螺旋结构具有稳定拉削力波 动的能力, 有利于拉削质量的提升; JIA 等 ${ }^{[7]}$ 对该种 拉刀的螺旋容屑槽刃磨方法进行了研究, 指出其成 功磨削的工艺保证约束, 并给出了基于包络原理的 砂轮安装位姿计算方法; JIA 等 ${ }^{[8-9]}$ 对该种螺旋内齿 圈拉刀切削齿的齿形磨削制造进行了研究, 优化了 集成四种切削齿形的磨削工艺路线, 并规划了相应 的磨削路径模式。尽管该种刀具的切削齿采用与传 统的环形容屑槽螺旋拉刀一致的螺旋渐开面结构, 然而其切削齿非对称的齿升量分布导致传统拉刀切 削齿磨削方法 ${ }^{[10]}$ 无法支持该种刀具精切齿的磨削, 需要针对该种刀具结构的开展面向磨削的数学模型 研究, 为其成功制造提供理论基础与技术支撑。

因此，本文在剖析螺旋内齿圈拉刀结构的基础 上, 构建精切齿位置分布的数学模型, 进而分析其 制造工艺性与加工质量保障要素, 进行高效的成型 磨削路径设计。在此基础上, 对精切齿侧面的完整 磨削且无干涉行程等问题进行研究, 并给出了面向
制造的砂轮优选模型。最终形成了面向螺旋内齿圈 拉刀精切齿高效精密磨削制造理论方法。

\section{2 拉刀结构与切削齿数学建模}

拉刀作为一种特种刀具, 依据工件目标加工结 构进行设计。为满足螺旋内齿圈的加工精度要求并 保证其拉削表面质量, 螺旋内齿圈拉刀在刀体上布 置不同齿形与切削余量的切削齿, 在一次拉削运动 中执行多阶段的切削工艺过程。因此, 要实现刀体 上各类切削齿的精密制造, 需要准确的构建出切削 齿的分布位置与齿升量模型, 为切削齿的数控磨削 实现提供支撑。

\section{1 切削齿坐标参数化建模}

螺旋内齿圈拉刀切削齿轴端面角度与目标内齿 圈保持一致的结构参数, 以确保加工中刀具相对内 齿圈毛胚做与其旋向及导程一致的螺旋运动能够准 确的切削出渐开线齿槽。同时, 为保证切削过程拉 削力的平稳与切屑的顺利排出, 螺旋内齿圈拉刀采 用端面均匀分布的螺旋容屑槽结构, 且其旋向与切 削齿旋向相反, 以形成如下优点。

(1) 螺旋容屑槽结构保证了切削齿连续性的切 入和切出齿圈, 有效地减小了拉削过程中整体切削 力的波动, 有助于提高齿圈的加工质量。

(2) 拉削属于封闭式切削, 螺旋容屑槽结构有 利于切屑的卷曲和排出, 有助于刀具使用寿命的 提升;

(3) 螺旋容屑槽的旋向与切削齿相反, 使得渐 开线廓型对应的切削齿左右两侧切削刃的前角调整 接近于正交切削, 相对于环型容屑槽极大的平衡了 两侧切削刃的切削力, 促进了齿圈螺旋渐开面的加 工精度与表面质量。

综合所述, 可知刀具结构参数包括切削齿结构 参数和容屑槽参数。其中, 切削齿结构参数包括: 导程 $L_{s}$, 螺旋角 $\beta_{p}$, 旋向 $\operatorname{Srot}($ 右旋 $=1$, 左旋 $=-1$ ), 端面模数 $m_{t}$, 齿数 $z$ 和端面压力角 $\alpha_{t}$ 等; 螺旋容屑 槽的结构参包括: 螺旋槽的旋向 $\operatorname{Grot}($ 右旋 $=1$, 左 旋 $=-1$ ), 螺旋角 $\beta_{g}$, 导程 $L_{g}$ 和螺旋槽的头数 $N_{g}$ 。

前述模型表明, 切削齿依据刀齿与容屑槽的螺 旋结构形成固定的双螺旋分布形式，因此对该模式 进行参数化表达, 以便于磨削加工中砂轮的运动坐 标计算。如图 1a 所示, 在刀具轴端面, 容屑槽结构 将刀体划分为 $N_{g}$ 个区间, 而切削齿槽又将每一区间 划分为 $N_{s}$ 个等份。值得注意, 每一螺旋槽划分区域 包含的切削齿槽数量 $N_{s}$ 不一定相等, 为便于程序控 制, 采用数组 $N s[]$ 顺序记录沿螺旋槽旋向方向的切 
削齿槽数 $N_{s}$ 。如此, 刀体上任一切削齿可由其所在 螺旋槽区域 $n_{g}$ 、齿形轨迹对应端面齿槽序号 $n_{s}$ 和齿 形轨迹上的轴向序号 $n_{p}$ 唯一确定: 设定一条螺旋容 屑槽最前端的切削齿为刀体表面切削齿分布网的原 点 $O_{s}=(0,0,0)$, 相应的每一切削齿在刀体切削齿分 布网络中对应坐标 $\left(n_{g}, n_{s}, n_{p}\right)$ 。由图 1a 可知, 原点 $O_{s}(0,0,0)$ 到切削齿 $D\left(n_{g}, n_{s}, n_{p}\right)$ 路径如下: 首先, 沿螺旋容屑槽移动 $n_{g}$ 个螺旋槽端面分割区间, 即对 应 $\sum_{i=0}^{n_{g}-1} N_{S}(i)$ 个齿槽; 然后, 沿切削齿轨迹回退 $n_{g}$ 个 切削齿位置, 即可移动到切削齿 $D$ 所在的容屑槽分 割区域 $N s\left(n_{s}\right)$ 的局部原点; 最后, 沿螺旋容屑槽移 动 $n_{s}$ 个齿槽, 并沿切削齿轨迹移动 $n_{p}$ 个切削齿位置, 即可到达 $D$ 齿。这种切削齿之间转换路径是确定切 削齿空间坐标的前提。

在切削齿的数控磨削中, 需要确定每一切削齿 在刀体(或机床)坐标系中的精确坐标位置, 这由切 削齿的位置参数与对应的分布网络坐标 $\left(n_{g}, n_{s}, n_{p}\right)$ 之 间的映射决定。如图 $1 \mathrm{~b}$ 所示, 螺旋内齿圈拉刀切削 齿遵循双螺旋分布模式, 即沿着切削齿轨迹的 $O_{s} A$ 和沿着螺旋容屑槽的 $O_{s} B$ 。为便于计算, 首先沿着 刀具回转轴将切削齿所处圆柱面展为一个平面, 然 后构建坐标系 $O_{s}-X_{s} A_{s}$, 其中 $X_{s}$ 与刀具轴线平行, $A_{S}$ 与 $X_{S}$ 垂直并指向表面展开的逆时针方向。这该坐 标系中, 切削齿的内在位置关系 $O_{s} A$ 和 $O_{s} B$ 分别表 达为 $\left(u p_{x}, u p_{a}\right)$ 和 $\left(u g_{x}, u g_{a}\right)$ 。基于正弦定理, 由 $\triangle O_{s} Q A$ 和 $\triangle O_{s} Q B$ 得结构参数如下

$$
\begin{aligned}
& \left(\begin{array}{c}
u p_{x} \\
u p_{a}
\end{array}\right)=\left(\begin{array}{l}
\frac{L_{g} \cdot \sin \left(\pi / 2-\beta_{g}\right) \cdot \cos \left(\beta_{p}\right)}{N_{s} \cdot \sin \left(\pi / 2+\beta_{g}-\beta_{p}\right)} \\
\frac{L_{g} \cdot \sin \left(\pi / 2-\beta_{g}\right) \cdot \sin \left(\beta_{p}\right)}{N_{s} \cdot \sin \left(\pi / 2+\beta_{g}-\beta_{p}\right)}
\end{array}\right) \\
& \left(\begin{array}{c}
X_{s} \\
A_{s}
\end{array}\right)=\left(\begin{array}{l}
X_{o}+u g_{x} \cdot\left(\sum_{t=0}^{n_{g}-1} N s t(t)+n_{s}\right)+u p_{x} \cdot\left(n_{p}-n_{g}\right) \\
A_{o}+G R o t \cdot u g_{a} \cdot\left(\sum_{t=0}^{n_{g}-1} N s t(t)+n_{s}\right)+P \operatorname{Rot} \cdot u p_{a} \cdot\left(n_{p}-n_{g}\right)
\end{array}\right)
\end{aligned}
$$

$$
\left(\begin{array}{l}
u g_{x} \\
u g_{a}
\end{array}\right)=\left(\begin{array}{c}
\frac{s_{n} \cdot \sin \left(\pi / 2-\beta_{p}\right) \cdot \sin \left(\beta_{g}\right)}{\cos \left(\beta_{p}\right) \cdot \sin \left(\pi / 2+\beta_{p}-\beta_{g}\right)} \\
\frac{s_{n} \cdot \sin \left(\pi / 2-\beta_{p}\right) \cdot \cos \left(\beta_{g}\right)}{\cos \left(\beta_{p}\right) \cdot \sin \left(\pi / 2+\beta_{p}-\beta_{g}\right)}
\end{array}\right)
$$

式中, $S_{n}=m_{n} \pi$, 为切削齿的法向弧齿厚。

假定切削齿分布网络原点切削齿 $O_{S}(0,0,0)$ 对 应的刀具表面坐标为 $\left(X_{o}, A_{o}\right)$, 综合刀齿分布网中的 切削齿转换路径与切削齿的位置关系参数, 即可计 算出切削齿坐标 $\left(n_{g}, n_{s}, n_{p}\right)$ 对应刀具表面坐标系的坐 标 $\left(X_{s}, A_{s}\right)$ 如下

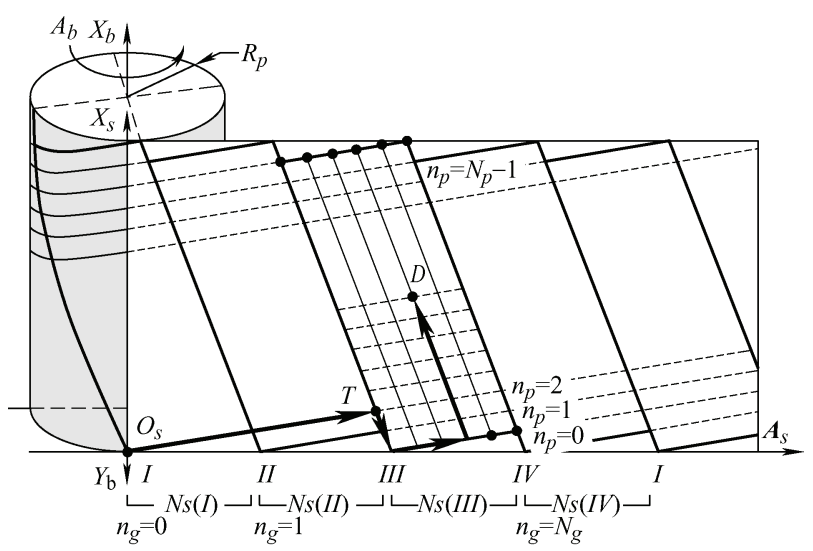

(a) 切削齿分布模式

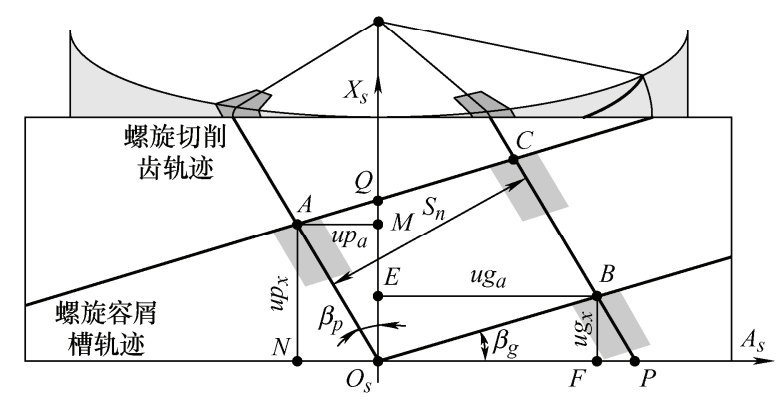

(b) 切削齿相对位置关系

图 1 螺旋内齿圈拉刀的切削齿坐标建模
面向磨削加工, 需要进一步将刀具表面坐标系 转换到刀具坐标系或机床坐标系。刀具坐标系 $O_{b} X_{b} Y_{b} Z_{b}$ 如图 1a 所示, $X_{b}$ 轴与刀具回转轴重合, $Y_{b}$ 轴通过刀具原点切削齿, $Z_{b}$ 轴由右手准则确定。进 一步指定绕刀具 $X_{b}$ 轴的回转运动为 $A_{b}$ 轴, 如此, 磨削中切削齿的定位可通过 $X_{b}$ 和 $A_{b}$ 轴的位移联动 控制实现, 对应坐标 $\left(X_{g}, A_{\mathrm{g}}\right)$ 计算如下

$$
\left(\begin{array}{c}
X_{g} \\
A_{g}
\end{array}\right)=\left(\begin{array}{cc}
1 & 0 \\
0 & R_{p}^{-1}
\end{array}\right) \cdot\left(\begin{array}{l}
X_{s} \\
A_{s}
\end{array}\right)
$$

式中, $R_{p}$ 为齿圈的分度圆半径。

\section{2 精切齿工作结构与齿升量模型}

螺旋内齿圈拉刀的精切齿段完成螺旋渐开面齿 形的精切, 并要求内齿圈 7 8 级的加工精度。因此, 执行齿形精切的一系列精切齿必须施加合理的切削 工作模式并匹配适当的齿升量, 这对精切齿的结构 提出了特殊的设计与制造要求。

遵循刀齿的双螺旋结构, 螺旋拉刀精切齿的结 构与工作示意如图 2 所示。由拉削的成型工作原理 
可知, 刀具精切段末端 $C-C^{\prime}$ 段对应的 $1 \sim 3$ 排切削 齿的齿形与齿圈目标渐开线廓型保持一致, 无齿升 量, 用于延长刀具的服役寿命和消除齿圈的回弹变 形。由于切削齿执行渐开线齿形的切削, 从制造经 济性角度考虑, 所有切削齿都采用一致的渐开线齿 形, 仅通过控制切削齿的法向弧齿厚 $s_{n, t}$ 变化来形成 相对齿升量, 可保证砂轮廓形的一致。与此同时, 螺旋容屑槽螺旋角一般小于切削齿的螺旋轨迹角, 所以精切齿两侧的切削刃前角分别形成锐角和针角 两种情形。通常锐角侧先执行切削, 即图中 $A^{\prime}-B^{\prime}$ 段的切削齿在锐角侧存在渐变的齿升量 $\Delta s_{n, t}$, 直至

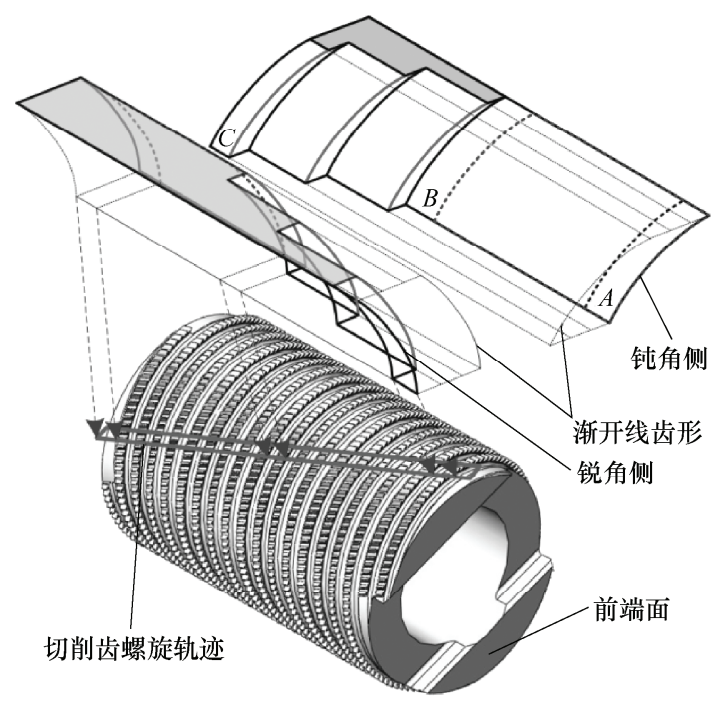

$B^{\prime}$ 对应廓型与 $C^{\prime}$ 廓型重合, 完成该侧齿圈对应渐 开线廓型的切削; 在这一过程中, 对应的 $A-B$ 段切 削齿形则保持不变, 无齿升量, 不参与切削但支撑 齿圈以保证拉削过程的平稳。随后, 切削齿两侧的 工作模式交换, $B-C$ 段切削齿侧存在渐变齿升量 $\Delta s_{n, t}$ 以完成该侧齿形的切削, 而 $B^{\prime}-C^{\prime}$ 侧齿形保持不变 引导刀具平稳完成拉削。有两点值得注意: 其一是 $A-A^{\prime}$ 段局部切削齿无齿升量, 保证齿圈从粗切平 稳过渡到精切; 其二是 $B-B^{\prime}$ 段局部切削齿亦无齿 升量, 保证齿圈不存在两侧同时切削情形发生, 保 证切削过程中每一时刻齿圈都具有基准。

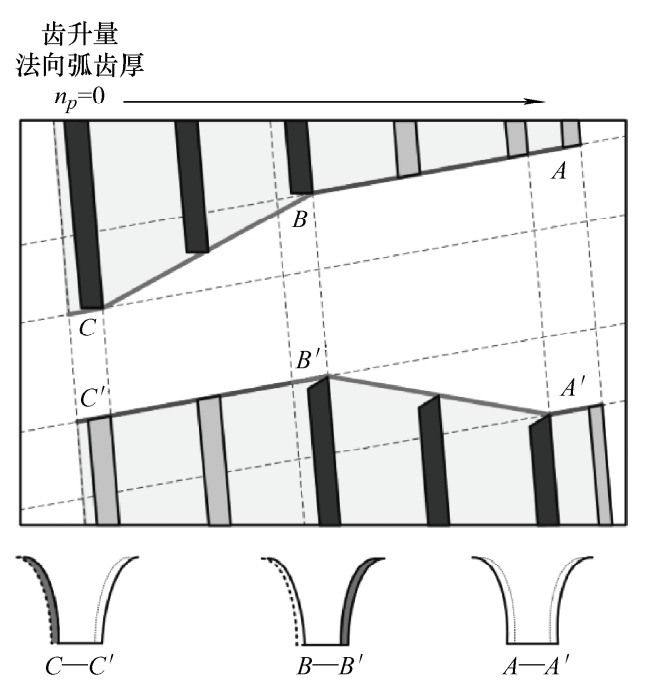

图 2 螺旋内齿圈拉刀精切齿结构与工作示意图

上述表明, 螺旋拉刀精切段切削齿的切削刃在 齿升量作用下, 呈现出一种非对称的台阶状模式。 为确保精切齿的精密磨削, 必须准确地描述精切齿 的齿升量。这里, 构建每一切削齿两侧的齿升量与 对应齿序号的关系如下: 指定精切段最前端切削齿 的序号 $n_{p}=0$ 并沿轴向向后连续增加切削齿的序号; 相应的锐角侧和钝角侧每齿齿升量, 即 $\Delta s_{n, t}$, 分别 顺序存储于数组 $S_{\text {acute }}$ ()和 $S_{\text {obtuse }}$ ()中, 数组的长度等 于精切齿的齿排数 $N_{p}$ 。

\section{3 精切齿磨削路径规划}

鉴于螺旋内齿圈拉刀精切齿的双螺旋分布模式 以及复杂齿升量分布, 需要综合考虑磨削制造效率、 制造可行性及制造精度保障等多种要素, 进行合理 的制造工序规划与磨削加工路径设计。

\section{1 精切段磨削工序规划}

\subsection{1 磨削工序的工艺性分析}

前述结构分析指出螺旋内齿圈拉刀的切削齿齿 形呈现出一种典型的非对称台阶模式。尽管每一刀
齿都为渐开线齿形, 然而每齿齿升量的精密要求和 海量的齿数对于精切齿的磨削工艺提出了挑战。这 里, 综合加工基准、磨削成型策略、精度检测和磨 削效率等要素对螺旋内齿圈拉刀精切齿的磨削工艺 性进行研究。

(1) 磨削加工基准。

拉刀的工作原理表明, 末端的切削齿的决定最 终的工件切削廓形, 而切削齿的齿升量为相对量并 决定着刀具的切削性能。因此, 以末端切削齿的齿 形为基准, 便于直接进行精度控制。

(2) 磨削成型方式。

切削齿待磨削的侧面为渐开螺旋面。鉴于切削 齿之间紧凑的空间, 采用展成磨削砂轮尺寸较大容 易引发干涉, 而且加工效率较低。因此采用砂轮成 型磨削较为合适。在螺旋结构成型磨削中, 砂轮两 侧磨削状态不相同, 会导致砂轮两侧磨损状态不同, 由此引发两侧表面磨削质量的不一致。同时, 切削 齿的齿升量随着切削齿轴向位置的不同而变换, 如 果采用砂轮双侧磨削, 则在磨削不同轴向位置的切 削齿时需要砂轮廓形需要相应的修瘦, 这将引入大量 
的砂轮修形操作从而导致加工效率低下, 并且导致砂 轮的利用率很低。因此, 采用砂轮单侧磨削的方式, 砂轮廓形固定, 仅在砂轮钝化后进行修锐即可。

(3) 加工精度控制。

精密加工中尺寸精度的控制至关重要。对于螺 旋齿槽结构, 采用跨棒距进行齿升量的检测较为合 适。尽管切削齿两侧齿升量的基准独立, 然而跨棒 距的检测无法与其基准发生关联, 即无法通过跨棒 距确定两侧齿升量的加工误差。因此, 对于切削齿 两侧齿升量分为两个阶段进行磨削, 待一侧完全磨 削完成并检验合格后, 进行另一侧齿升量的磨削。 如此跨棒距检测的误差对应唯一齿侧, 进而可进行 加工调整确保精度。

(4) 磨削效率提升。

前述分析指出, 采用砂轮双侧磨削会引发频繁 的砂轮修形操作, 严重制约加工效率。采用砂轮单 侧磨削时, 切削齿两侧分为两个阶段磨削, 并进行 误差修正。切削齿锐角侧齿升量位于拉刀精切段的 前段, 因此锐角侧的总磨削行程小于针角侧磨削行 程。因此, 先加工针角侧, 当钝角侧出现整体性齿 升量偏差时, 通过对锐角侧齿升量进行修正进行补 偿即可, 磨削时间短, 利于提升加工效率。

\subsection{2 磨削工序规划}

基于上述工艺分析与对比，规划螺旋内齿圈拉 刀精切齿的加工为三个阶段, 如图 3 所示, 依次为 校准齿齿形基准磨削、针角侧齿升量磨削和锐角侧 齿升量磨削，具体如下。

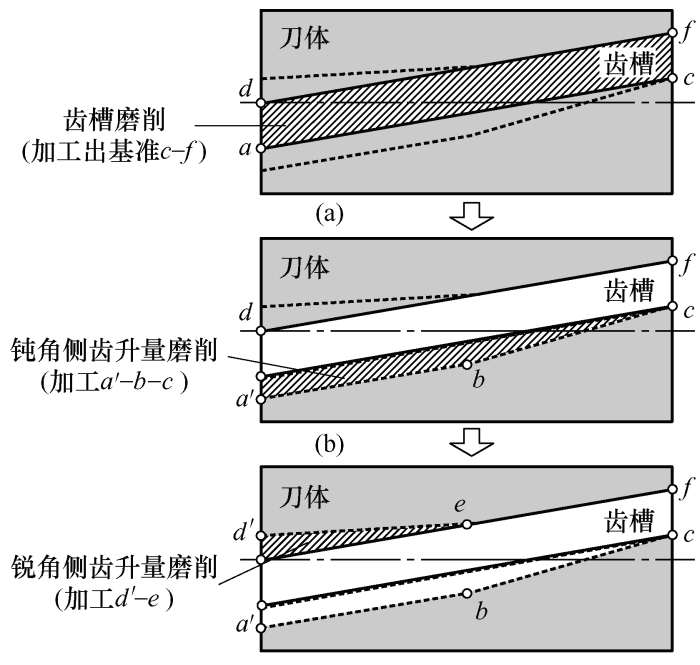

(c)

图 3 螺旋内齿圈拉刀精切齿的磨削流程划分

(1) 以拉刀校准齿廓形为对象, 加工贯穿刀体 的齿槽, 磨削行程为图 $3 \mathrm{a}$ 中的 $a-c$ 和 $d-f$, 最终完成 整个切削齿槽基准的加工。

(2) 以锐角侧齿面 $d-f$ 为基准, 对齿槽中钝角侧
切削刃的齿升量进行磨削, 即磨削图 $3 \mathrm{~b}$ 中 $a^{\prime}-b-c$ 的 台阶型切削刃结构。

(3) 以钝角侧齿面 $a^{\prime}-b$ 为基准, 对齿槽中锐角 侧切削刃的齿升量进行磨削, 即磨削图 $3 \mathrm{c}$ 中 $d^{\prime}-e$ 的 台阶型切削刃结构。

在每一磨削阶段, 通过跨棒距进行齿形和齿升 量精度的控制。

\section{2 磨削路径规划}

\subsection{1 磨削路径规划要素分析}

基于前述磨削工序，在进行螺旋内齿圈拉刀精 切齿磨削路径规划时, 需要针对精切齿的非对称台 阶齿升量结构, 从切削齿表面的磨削质量和磨削效 率进行重点考虑。

（1）切削齿结构一致性。

对于螺旋内齿圈拉刀而言，在拉削过程中，整 体齿槽的切削齿相同轴向位置的切削齿同时参与切 削, 如果不同齿槽之间的拉削状态将存在较大差异, 不仅导致齿圈齿性表面拉削质量的波动，甚至造成 局部切削齿的损坏导致刀具报废。由此可见，精切 齿的磨削保证全体齿槽切削齿齿升量的一致非常重 要。同时就精切齿轴向的齿升量而言, 即使齿升量 存在误差, 只要全体齿槽的齿升量保持一致, 也可 以保证拉削过程稳定，后续通过调节磨削余量可进 行齿升量的调整。因此，对于每一个齿升量磨削操 作，待其完成刀体全体齿槽磨削后，进行下一个吃 刀量的磨削加工。这样，一方面可保证全体齿槽中 切削齿齿升量的一致性，另一方面可保证全体齿槽 跨棒距检测结果理论上相同, 有利于齿形精度控制。

(2) 磨削表面质量一致性。

磨削中砂轮廓形的即时修锐有助于保证切削齿 的表面磨削质量与齿形精度。在精切齿齿升量磨削 中, 砂轮对切削齿单侧表面进行磨削。如果每一磨 削行程中砂轮的吃刀量不一致, 一方面会造成切削 齿的磨削工作状态波动，难以维持切削齿表面磨削 质量的稳定性, 另一方面难以评估砂轮的磨损程度, 从而无法有效设置砂轮廓形修锐操作的自动执行。 因次, 磨削中砂轮采用相同的进给量, 能够在保证 切削齿表面质量与磨削精度稳定的同时, 便于依据 磨削总行程估算砂轮的磨损程度, 有助于砂轮修锐 的自动化以提升加工效率。

(3) 磨削效率提升。

在磨削过程中，频繁的加减速运动以及过多的 磨削空行程都会严重降低加工效率。针对精切齿的 台阶型齿升量结构, 应尽可能的延长每一磨削行程 的有效长度, 以减小行程始末加减速运动的代价, 同时尽可能缩短无效磨削行程, 减少无效加工时间。 
因此, 从刀具磨削材料去除角度考虑, 将刀具前端 至切削齿每一齿升量轴向位置的范围设置为对应的 磨削行程, 可确保无磨削空行程且路径最大化。在 此基础上, 沿切削齿齿升量台阶深度方向逐步施加 吃刀量, 重复构建磨削行程。

(4) 磨削定位精度保障。

在切削齿齿升量的精密磨削中, 必须保证砂轮 相对于拉刀的运动精度、定位精度以及重复定位精 度等。因此, 在磨削路径的每一进刀位置处应增加 高精度的准停功能, 并对螺旋面的加工通过数控系 统施加轨迹廓形误差控制, 具体操作在数控编程中 通过配置机床功能参数完成。

\section{2 .2 单齿槽磨削路径模式}

基于上述分析，规划单个齿槽精切齿齿升量的 砂轮磨削走刀路径如图 4 所示。以齿槽中心在刀具前 端面外的一点 $O$ 为局部原点, 作为砂轮的进退刀参考 点。以钝角侧磨削为例, 操作步骤简化描述如下。

(1) 砂轮运动到齿槽的坐标原点 $O$ 。

(2) 绕刀具轴线旋转施加设定吃刀量 $F_{1}$ 。

(3) 砂轮按照切削齿的螺旋轨迹执行磨削行程 $T_{1}$, 直至轴向最后一齿 $N_{e}{ }^{o b}$ 前方停止, 随后按原路 径退回行程起点。重复施加齿升量并执行往复螺旋 磨削行程, 直至齿 $N_{e}{ }^{o b}$ 对应的齿升量完全加工。

(4) 切换到下一齿槽, 重复步骤(2)和(3), 直至 所有齿槽完成磨削。

(5) 依据步骤(2)、3 和(4), 施加吃刀量 $F_{2}$, 对 第 $N_{e}^{o b}-1$ 齿的齿升量进行磨削, 相应的磨削行程也 缩短为 $T_{2}$ 。如此重复, 磨削行程随齿升量台阶结构 逐渐缩短, 直至完成第 $N_{s}^{o b}$ 齿的齿升量磨削, 从而 完成针角侧齿升量的磨削。

同样的, 锐角侧齿升量的磨削也按照同样的方 式从第 $N_{e}^{a c}$ 齿到第 $N_{s}^{a c}$ 齿之间执行。

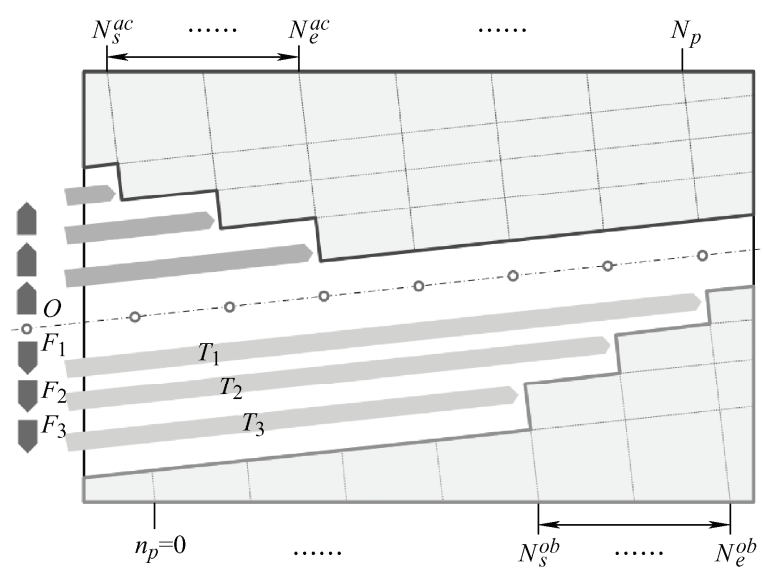

图 4 精切齿齿升量磨削路径示意图

对于第 $n_{g}$ 齿槽第 $n_{s}$ 个切削齿的磨削行程, 其起
始和终止位置的坐标, 分别按照第 $\left(n_{g}, n_{s}, 0\right)$ 和第 $\left(n_{g}\right.$, $n_{s}, n_{p}$ ) 个齿的坐标通过式(3)计算。在施加切削齿法向 弧齿厚方向的磨削进给量 offset 后, 对行程坐标的 回转轴坐标施加对应方向的偏执 $A_{\text {off }}$

$$
A_{\text {off }}= \pm \frac{\text { offset }}{\cos \left(\beta_{\mathrm{s}}\right) R_{p}}
$$

值得注意, 这里的路径仅为砂轮的运动模式, 对于每一轴向切削齿的具体磨削行程, 在后续内容 中进行深入研究。

\subsection{3 磨削路径流程}

基于前述分析, 设计螺旋内齿圈拉刀精切齿的 完整磨削流程如图 5 所示。

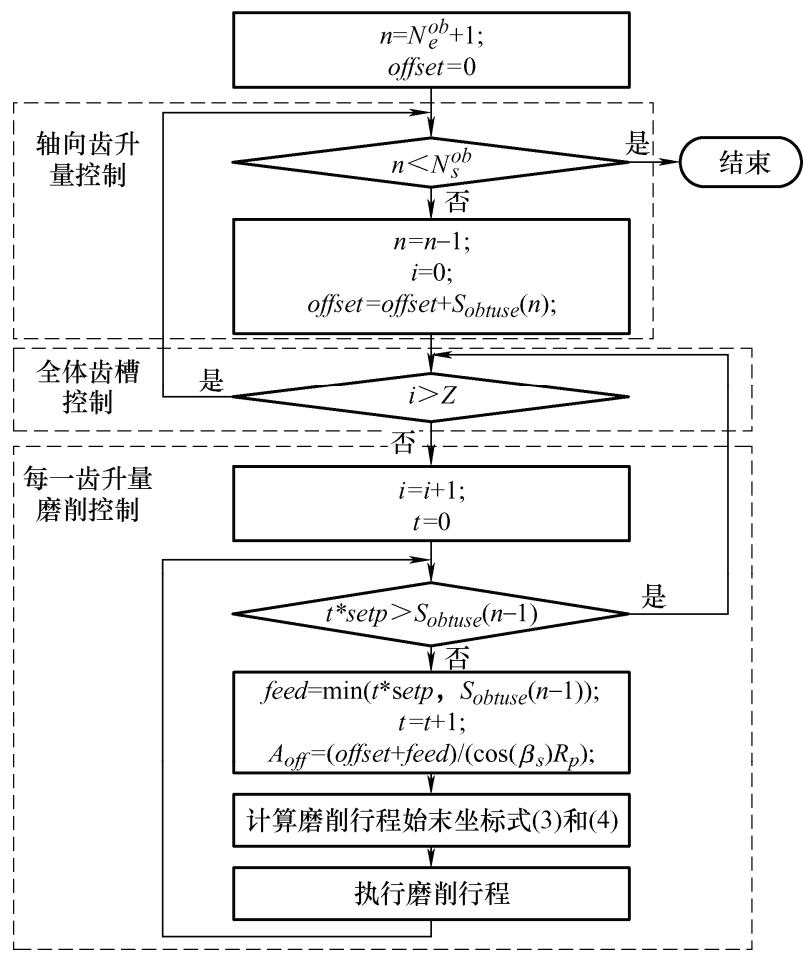

图 5 螺旋内齿圈拉刀切削刃齿升量磨削操作流程

首先, 初始化齿槽齿升量的磨削序号 $n$ 等于最 末段的齿序号 $N_{e}^{o b}+1$, 并设置每一齿升量磨削的砂 轮累计进刀偏移量 offset $=0$ 。

然后, 判断所有齿升量是否都得到磨削: 是, 结束退出; 否, 进入前一齿升量的磨削循环中, 并 进行齿槽控制变量 $i$ 的初始化与砂轮累计进刀量偏 移 offset 的累加。

接下来, 判断整圈齿槽是否都得到磨削: 是, 返回所有齿升量完成判定环节; 否, 对当前第 $i+1$ 齿槽的第 $n-1$ 排齿对应齿升量进行处理，具体如下 所述。

首先, 判定当前施加的砂轮进刀量 $t * f e e d$ 是否 大于第 $n-1$ 排齿的齿升量 $S_{\text {obtuse }}(n-1)$ : 大于等于表 明当前齿升量 $(i$ 齿槽 $n$ 排齿升量)加工完成，退出当 
前操作; 小于表明当前齿升量尚未完全磨削, 确定 当前的砂轮吃刀量 $f e e d=\min \left(t^{*}\right.$ step, $\left.S_{\text {obtuse }}(n-1)\right)$, 即 保证总的吃刀量不超出当前齿升量, 其中 step 为设 定的砂轮单位吃刀量。同时, 依据式(5)计算当前砂 轮的进给量, 并通过式(3)和(4)计算当前磨削行程的 起始和终止位置坐标, 然后执行一次往磨削行程。 随后, 跳转回当前齿槽齿升量加工完成判断。

\section{4 精切齿磨削行程计算}

螺旋内齿圈精切齿的齿升量呈台阶形, 砂轮需 要在对切削齿侧面进行完整磨削的同时, 不能与相 邻切削齿发生干涉, 破坏刀齿结构。在第 3 节中规 划的磨削路径仅为路径运动模式, 需要在磨削行程 末端进一步保证切削齿侧面的无干涉完整磨削。因 此, 合理的切削齿侧磨削行程设置与砂轮选择是保 证拉刀精切齿成功磨削的前提。

\section{1 砂轮廓形计算}

在切削齿面的成型磨削中, 砂轮与拉刀的安装 位置如图 6 所示, 砂轮回转轴 $Z_{g}$ 与刀具回转轴 $Z_{b}$ 之间的距离为 $A, Y_{w}$ 轴与 $Z_{b}$ 轴的夹角为 $\Sigma$, 砂轮绕 刀具轴线 $Z_{b}$ 做与切削齿导程 $L_{s}$ 一致的螺旋运动, 完 成切削齿螺旋侧面的磨削。
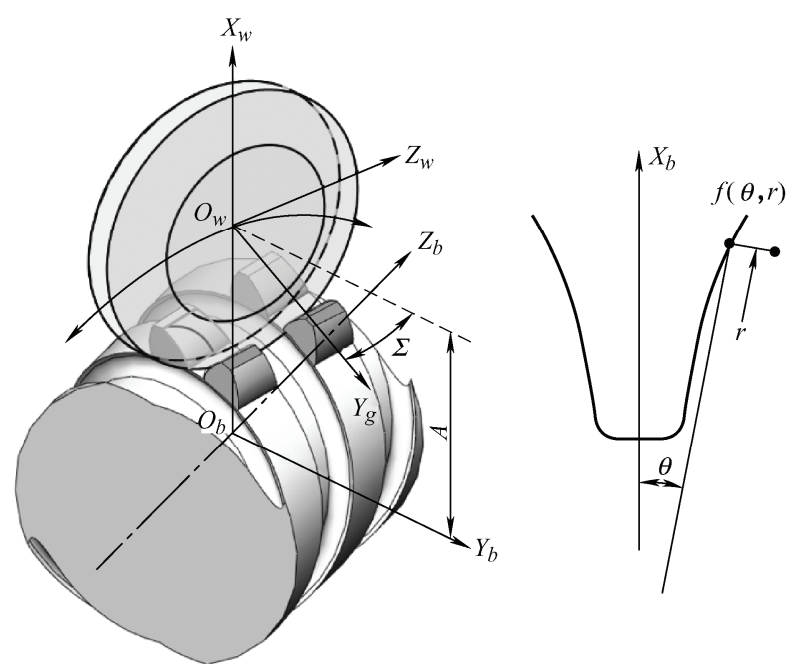

图 6 精切齿磨削砂轮廓形计算

由于切削齿的端面廓形为渐开线, 可知廓形上 一点为关于滚动角 $\theta$ 的函数 $\boldsymbol{f}_{b}(\theta)=\left[x_{b}(\theta), y_{b}(\theta), 1\right]^{\mathrm{T}}$

$f_{b}(\theta)=\left(\begin{array}{c}x_{b}(\theta) \\ y_{b}(\theta) \\ 1\end{array}\right)=\left(\begin{array}{c}r_{b} \cos (\theta+\xi)+r_{b} \sin (\theta+\xi) \theta \\ \pm\left(r_{b} \sin (\theta+\xi)-r_{b} \cos (\theta+\xi) \theta\right) \\ 1\end{array}\right)$

式中, $\theta \in\left[\tan \left(\cos ^{-1}\left(r_{b} / r_{f}\right)\right), \tan \left(\cos ^{-1}\left(r_{b} / r_{a}\right)\right)\right] ; r_{b}$ 为 端面基圆半径; $r_{f}$ 为齿根高半径; $r_{a}$ 为齿顶高半径; $\xi$ 为渐开线起点与 $Y_{b}$ 轴夹角, 有 $\xi=\pi(2 Z)^{-1}-$ $\left(\tan \left(\alpha_{t}\right)-\alpha_{t}\right) ; \pm$ 表示两侧廓形。

切削齿侧面 $\boldsymbol{S}_{b}(\theta, \varphi)$ 由端面廓形 $\boldsymbol{f}_{b}(\theta)$ 绕 $Z_{b}$ 轴旋转 $\varphi$ 生成

$$
\boldsymbol{S}_{b}(\theta, \varphi)=\left(\begin{array}{c}
x_{b}(\theta) \cos (\varphi)-y_{b}(\theta) \sin (\varphi) \\
x_{b}(\theta) \sin (\varphi)+y_{b}(\theta) \cos (\varphi) \\
\operatorname{Srot}_{s} \varphi(2 \pi)^{-1}
\end{array}\right)
$$

由空间包络原理可知, 在磨削中, 砂轮外表面 与切削齿螺旋面在切触点 $\boldsymbol{P}=[x, y, z]^{\mathrm{T}}$ 处接触且存在 公法线。由于砂轮表面任意点的法线通过砂轮回转 轴 $\boldsymbol{Z}_{w}=[0, \cos (\Sigma),-\operatorname{Srotsin}(\Sigma)]^{\mathrm{T}}$, 所以螺旋面在切触点 $\boldsymbol{P}$ 的法线 $\boldsymbol{N}_{P}$ 和砂轮回转轴 $\boldsymbol{Z}_{w}$ 及 $\boldsymbol{O}_{w} \boldsymbol{P}$ 共面, 即有

$$
\boldsymbol{O}_{w} \boldsymbol{P} \cdot\left(\boldsymbol{N}_{p} \times \boldsymbol{Z}_{w}\right)=0
$$

其中, $\boldsymbol{O}_{w}=[0,0, A]^{\mathrm{T}} ; \boldsymbol{N}_{p}(\theta, \varphi)$ 通过微分几何确定

$$
\boldsymbol{N}_{p}(\theta, \varphi)=\frac{\frac{\partial \boldsymbol{S}_{b}(\theta, \varphi)}{\partial \theta} \times \frac{\partial \boldsymbol{S}_{b}(\theta, \varphi)}{\partial \varphi}}{\left|\frac{\partial \boldsymbol{S}_{b}(\theta, \varphi)}{\partial \theta} \times \frac{\partial \boldsymbol{S}_{b}(\theta, \varphi)}{\partial \varphi}\right|}
$$

由于螺旋渐开面是关于 $\theta$ 和 $\varphi$ 的双变量函数, 当给定转角 $\theta$ 即可确定唯一的半径 $r$, 式(7)变为关 于转角 $\varphi$ 的单变量方程。通过数值搜索算法确定 $\varphi$, 即可得到切削齿表面半径为 $r$ 处的切触点坐标。对 切削齿径向范围及逆行离散, 计算各半径处的切触 点, 得到切削齿螺旋面的磨削接触线 $\boldsymbol{C}=\left[x_{c}, y_{c}, z_{c}\right]^{\mathrm{T}}$ 。 然后, 将该磨削接触线每一点转换到砂轮坐标系中, 再绕 $Z_{g}$ 轴旋转使之投影到 $X_{w}-O_{w}-Z_{w}$ 平面, 得到砂 轮廓形曲线一点 $\boldsymbol{f}_{G}=\left[x_{g}, y_{g}, z_{g}\right]^{\mathrm{T}}$ 如下

$$
\left[\begin{array}{c}
x_{g} \\
y_{g} \\
z_{g}
\end{array}\right]=\left(\begin{array}{c}
\sqrt{\left(x_{c}-A\right)^{2}+\left(y_{c} \sin (\Sigma)-z_{c} \cos (\Sigma)\right)^{2}} \\
0 \\
y_{c} \cos (\Sigma)+z_{c} \sin (\Sigma)
\end{array}\right)
$$

\section{2 切削齿磨削行程建模与计算}

要保证台阶型齿升量结构精切齿的精确磨削加 工, 不仅要完整的磨削切削齿侧面, 还需要避免砂 轮与后一齿切削刃的干涉。此外, 每一磨削行程需 要保证一定的砂轮磨削越程量, 以对砂轮的对刀误 差等进行包容。

\subsection{1 精切齿侧面完全磨削行程计算}

前述成型磨削砂轮廓形计算表明, 砂轮通过磨 削接触线 $\boldsymbol{C}$ 扫掠出切削齿侧面。因此, 要保证切削 齿侧面完全磨削, 如图 7 所示, 砂轮的磨削接触线 $C$ 沿拉刀轴线方向的运动需要完整覆盖切削齿的前 刃曲线 $\boldsymbol{E}_{f}$ 和齿背刃曲线 $\boldsymbol{E}_{b}$ 。由此确定出最小磨削行 程为 $S_{0}$ 至 $E_{0}$ 。其中, $S_{0}$ 为砂轮磨削接触线 $\boldsymbol{C}$ 完全 位于切削刃 $\boldsymbol{E}_{f}$ 前方并保持点接触的轴向位置; $E_{0}$ 为砂轮磨削接触线 $\boldsymbol{C}$ 完全位于齿背曲线 $\boldsymbol{E}_{b}$ 后方并 
保持点接触的轴向位置; $f_{1}$ 和 $f_{2}$ 分别为砂轮在切削 齿前方和后方的磨削越程量。

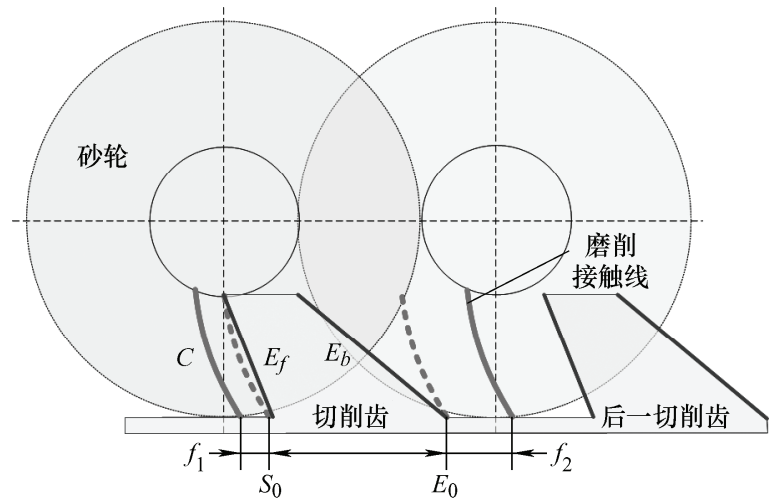

图 7 切齿齿齿侧完整磨削行程分析

切削齿左右侧的切削刃曲线 $\boldsymbol{E}_{f}^{L}$ 和 $\boldsymbol{E}_{f}^{R}$ 与左右侧 齿背曲线 $\boldsymbol{E}_{b}{ }^{L}$ 和 $\boldsymbol{E}_{b}{ }^{R}$, 通过对螺旋前刀面(后刀面)与 切削齿侧面求交确定。然后, 依据磨削接触线 $\boldsymbol{C}$ 包 含的离散点, 逐一计算对应的切削刃点和齿背曲线 点坐标。由此, 通过全体离散点确定出在刀具坐标 系中切削齿侧面完整磨削的砂轮轴向运动位置 $S_{0}$ 和 $E_{0}$ 分别如下

$$
\begin{aligned}
& S_{0}=\min \left(\boldsymbol{E}_{f} \cdot[0,0,1]^{\mathrm{T}}-\boldsymbol{C} \cdot[0,0,1]^{\mathrm{T}}\right) \\
& E_{0}=\min \left(\boldsymbol{E}_{b} \cdot[0,0,1]^{\mathrm{T}}-\boldsymbol{C} \cdot[0,0,1]^{\mathrm{T}}\right)
\end{aligned}
$$

由于砂轮两侧磨削接触线不对称, 且切削齿两 侧切削刃曲线和齿背曲线也不相同, 因此需要对精 切齿两侧分别进行完整磨削行程的计算。

\subsection{2 齿侧无干涉磨削行程计算}

精切齿台阶型齿升量要求, 砂轮对切削齿侧面 进新磨削时, 不能与后一切削齿的切削刃发生干涉。 因此, 需要确定砂轮与后一切削齿切削刃接触的极 限位置。加工中砂轮沿切削齿的螺旋特征进行走刀, 如图 8 所示, 后一齿切削刃 $\boldsymbol{E}_{f}$ 与砂轮表面最先发生 接触于点 $\boldsymbol{M}$, 则点 $\boldsymbol{M}$ 到点 $\boldsymbol{P}$ 之间的距离 $\mid \boldsymbol{P M}$ 即为 当前侧切削刃在刀具坐标系中沿轴向的最大无干涉 磨削行程, 确定如下

$$
|\boldsymbol{P M}|=\min \left(\boldsymbol{E}_{f} \cdot[0,0,1]^{\mathrm{T}}-\boldsymbol{M} \cdot[0,0,1]^{\mathrm{T}}\right)
$$

从数值计算角度出发, 对砂轮与切削刃的接触 点 $\boldsymbol{M}$ 进行求解。如图 8 所示, 点 $\boldsymbol{M}$ 和 $\boldsymbol{N}$ 是切削刃 上一点 $\boldsymbol{P}=\left[x_{P}, y_{P}, z_{P}\right]^{\mathrm{T}}$ 沿磨削的螺旋轨迹距离砂轮表 面 $\boldsymbol{S}_{G}$ 为 0 的点, 其满足如下方程

$$
f(t)=\boldsymbol{M}_{S}(t) \cdot \boldsymbol{P}-\boldsymbol{S}_{G}=0
$$

式中, 砂轮表面 $\boldsymbol{S}_{G}$ 由砂轮廓形 $\boldsymbol{f}_{G}$ 绕回转轴旋转 $360^{\circ}$ 确定; $\boldsymbol{M}_{S}(t)$ 为磨削运动矩阵, 具体如下

$$
\boldsymbol{M}_{S}(t)=\left[\begin{array}{ccc}
\cos \left(2 \pi t / L_{s}\right) & -\sin \left(2 \pi t / L_{s}\right) & 0 \\
\sin \left(2 \pi t / L_{s}\right) & \cos \left(2 \pi t / L_{s}\right) & 0 \\
0 & 0 & t
\end{array}\right]
$$

对于切削刃每一离散点构建方程式(14), 并可 通过数值搜索算法进行求解。实际中, 如图 8 所示, 可能存在两个解 $\boldsymbol{M}$ 和 $\boldsymbol{N}$, 其中 $t$ 值小的点为真解。 最终, 将完整切削刃的计算结果代入式(12), 即可 得到砂轮与后一切削齿切削刃无干涉的最大砂轮磨 削行程。实际操作中, 应进一步匹配合理的砂轮越 程量进行设置。

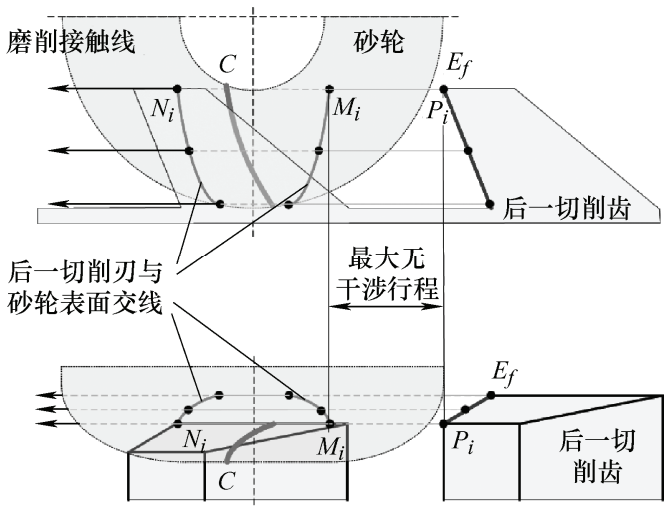

图 8 切削齿无干涉磨削行程分析

\subsection{3 切削齿磨削行程集成建模}

综合切削齿侧面的完整磨削与无干涉条件, 在 拉刀坐标系中, 砂轮对于每一切削齿轴向的局部磨 削行程 $\left[T_{s}, T_{e}\right]$ 必须满足式(16), 即磨削行程起始轴向 位置 $T_{s}$ 小于切削齿完全磨削的起始位置 $S_{0}$, 并保证 一定的砂轮越程量 $f_{1}$; 同时, 磨削行程的轴向停止 位置大于切削齿侧面完全磨削的终止位置 $E_{0}$, 并保 证一定砂轮越程量 $f_{2}$, 而且不能够超过砂轮最大无 干涉行程 $|\boldsymbol{P M}|$ 。

$$
\left\{\begin{array}{l}
T_{s} \leqslant\left(S_{0}-f_{1}\right) \\
\left(E_{0}+f_{2}\right) \leqslant T_{e} \leqslant|\boldsymbol{P M}|
\end{array}\right.
$$

上述磨削行程条件需要针对切削齿两侧分别进 行计算与校核。在磨削操作中, 强烈建议精切齿两 侧的磨削采用统一的对刀点, 便于维护砂轮与切削 齿两侧螺旋渐开面之间的位置关系。

\section{3 面向磨削工艺的砂轮选型与安装优化}

在精切齿齿形磨削中, 当砂轮对刀点设定, 后 续各磨削工序中都不应再进行改动以避免引入额外 的对刀加工误差, 造成砂轮与切削齿之间的相对位 置带来不确定。实际操作中, 一般选用大半径的砂 轮, 以延长砂轮使用寿命, 减少砂轮的更换次数、 缩短工作准备时间, 并回避磨削过程中由于更换砂 轮引入的加工误差等。

砂轮廓形计算模型指出, 砂轮的安装角 $\Sigma$ 和安 装中心距决定了砂轮与切削齿表面之间磨削接触线 形态, 进而间接地决定了不等式(16)所示的切削齿 磨削行程。因此, 将砂轮半径看作关于砂轮安装中 
心距 $A$ 和砂轮安装角 $\Sigma$ 的函数 $R(A, \Sigma)$ 。在此基础上, 可构建出在式(15)约束条件下最大化砂轮半径的优 化问题如下

$$
\begin{array}{ll}
\max & R(A, \Sigma) \\
\text { s.t. } & T_{s} \leqslant\left(S_{0}-f_{1}\right) \\
& \left(E_{0}+f_{2}\right) \leqslant T_{e} \leqslant|\boldsymbol{P M}|
\end{array}
$$

该问题为一个双变量约束优化问题, 可通过数 值搜索方法得到最大的可行砂轮半径 $R_{\text {max }}$ 与对应的 安装角 $\Sigma$ 。

\section{5 实例验证与分析}

为表明本文提出方法的有效性, 以一把螺旋内 齿圈拉刀精切齿的磨削为例进行加工参数的计算与 验证。拉刀的主要结构参数如表 1 所示。

表 1 螺旋内齿圈拉刀参数

\begin{tabular}{lc}
\hline \multicolumn{1}{c}{ 参数 } & 数值 \\
\hline 容屑槽参数 & \\
导程 $L_{g} / \mathrm{mm}$ & 50.8 \\
头数 $N_{s}$ & 4 \\
旋向 $G r o t$ & -1 \\
前角 $\left./{ }^{\circ}\right)$ & 18 \\
齿背角 $\left./{ }^{\circ}\right)$ & 40 \\
齿顶厚 $/ \mathrm{mm}$ & 4.6 \\
容首槽深度 $/ \mathrm{mm}$ & 5.6 \\
齿形参数 & \\
齿数 $Z$ & 98 \\
端面模数 $m_{t} / \mathrm{mm}$ & 1.4932 \\
端面压力角 $\alpha_{t} /\left(^{\circ}\right)$ & 20.4479 \\
螺旋角 $\left.\beta_{s} /{ }^{\circ}\right)$ & 22.556 \\
旋向 $S r o t$ & 1 \\
齿升量参数 & \\
左侧最大齿升量 $\Delta S_{n} / \mathrm{mm}$ & \\
右侧最大齿升量 $\Delta S_{n} / \mathrm{mm}$ & 0.024 \\
\hline
\end{tabular}

\section{1 磨削加工路径}

对表 1 所示螺旋内齿圈拉刀精切段, 按照式(1) 和(2)计算出基本参数 $\left[u p_{x}, u p_{a}\right]=[12.1429,5.0437]$, $\left[u g_{x}, u g_{a}\right]=[0.4956,4.4871]$ 。

基于本文提出的精切齿齿升量磨削路径规划方 法, 程序化生成路径如图 9 所示: 每一切削齿槽的 前段, 切削齿锐角侧齿形的磨削路径依照切削齿的 齿升量呈对应的台阶结构; 每一齿槽的整段, 针角 侧的磨削路径形成与齿升量一致的台阶结构; 相邻 齿槽之间在齿槽的最前端沿着容屑槽的轨迹进行磨 削任务的切换。

\section{2 磨削行程计算实例}

选择安装角 $\Sigma=22.56^{\circ}$ 砂轮安装中心距 $A=$ $80 \mathrm{~mm}$, 进行精切齿磨削行程的分析与计算。令在齿 顶圆上，齿槽中心轨迹与螺旋前刀面的交点为刀具坐 标系原点。以该点为砂轮设置的轴向原点, 该拉刀精 切齿的磨削轴向行程范围计算结果如表 2 所示。

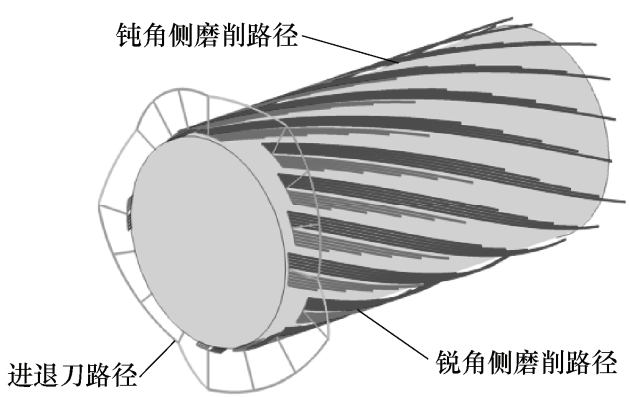

图 9 螺旋内齿圈拉刀精切齿磨削路径

表 2 精切齿磨削行程

\begin{tabular}{cccc}
\hline \multirow{2}{*}{ 齿侧 } & 完整磨削起点 & $\begin{array}{c}\text { 完整磨削终点 } \\
S_{0} / \mathrm{mm}\end{array}$ & $\begin{array}{c}\text { 最大无干涉位置 } \\
E_{0} / \mathrm{mm}\end{array}$ \\
\hline 锐角侧 & 3.5004 & 9.4556 & $1 \mathrm{~mm}$ \\
针角侧 & -4.1315 & 4.5774 & 5.7923 \\
\hline
\end{tabular}

精切齿针角侧和锐角侧齿形的磨削行程计算模型 分别如图 10a 和 $10 \mathrm{~b}$ 所示: 在轴向最前方位置 $S_{0}$ 处砂 轮表面磨削接触线 $\boldsymbol{C}$ 完全位于切削刃曲线 $\boldsymbol{E}_{f}$ 的前方, 并与切削刃在顶端发生点接触; 在轴向中间位置 $E_{0}$ 处 的砂轮表面磨削接触线 $\boldsymbol{C}$ 完全位于切削刃齿背曲线 $\boldsymbol{E}_{b}$ 后方, 并与齿背曲线 $\boldsymbol{E}_{b}$ 在齿根位置处接触; 在轴向最 后方位置处, 砂轮表面与后一切削齿的切削刃顶部接 触, 即将发生干涉; 砂轮的磨削越程量 $f_{2}$ 不能超出砂 轮从中间位置 $E_{0}$ 到与后一切削齿发生接触位置之间的 距离。由图 10 可清晰得知, 当前砂轮能够完成对切削 齿锐角侧和针角侧的完整磨削, 而且与后一切削齿的 切削刃不发生干涉，并能够施加一定的磨削越程量。

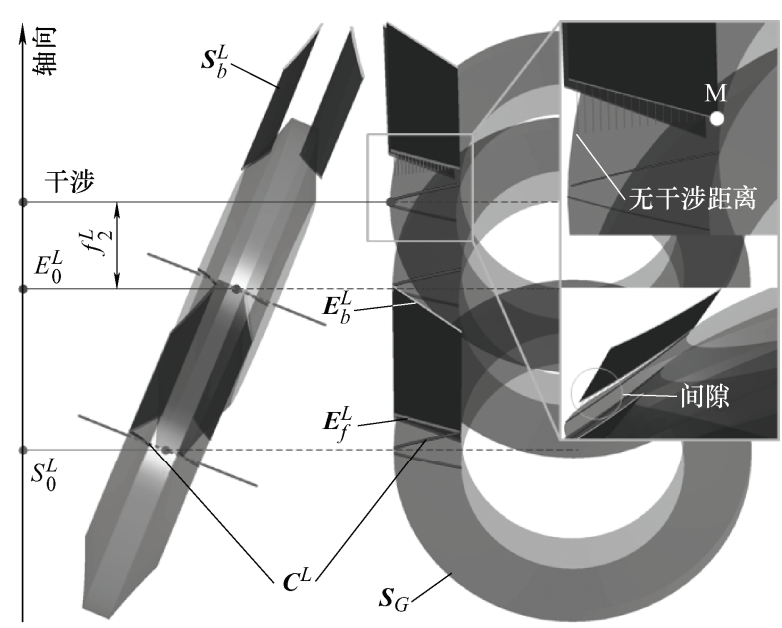

(a) 钝角侧磨削行程 


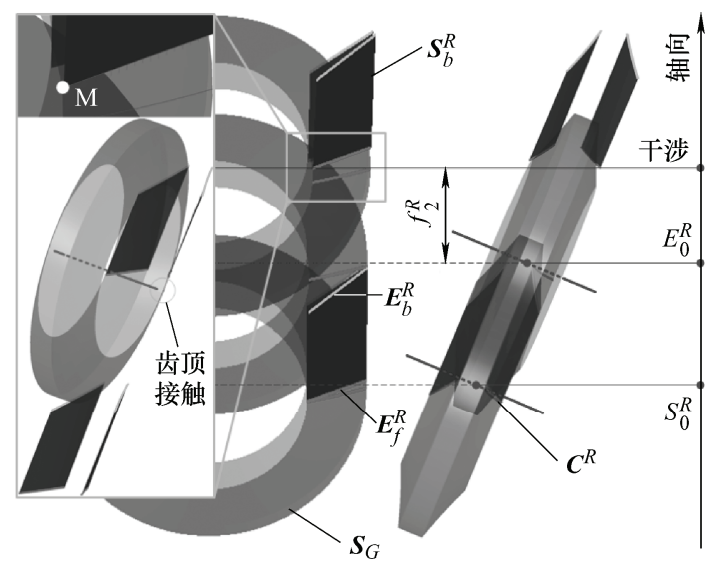

(b) 锐角侧磨削行程

图 10 螺旋拉刀精切齿磨削行程

采用万能数控拉刀磨床 QMK008 对该螺旋拉 刀精切齿段毛坏样件进行磨削。为突出对于每一精 切齿侧面的行程精确控制, 进行齿侧的铲背磨削。 磨削结果如图 11a 所示: 齿侧面后部磨亮, 表明对 切削齿形成了铲背修瘦; 而切削齿前部保留涂色, 表明砂轮未与之发生干涉。同时, 图 $11 \mathrm{~b}$ 为采用机 床测头对一齿槽内所有切削齿齿升量的测量结果, 表明本文磨削路径能够实现该种螺旋内齿圈拉刀非 对称台阶型齿升量的磨削。
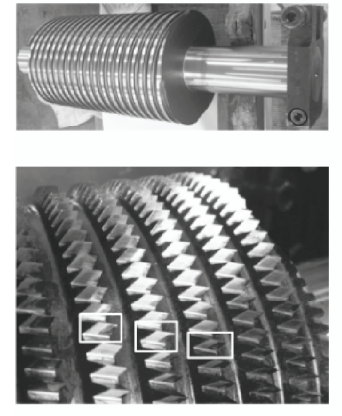

(a) 毛坯与磨削样件

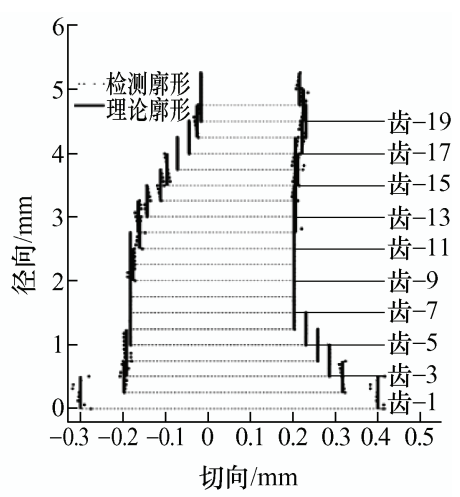

(b) 切削齿螺旋线检测形态
图 11 螺旋内齿圈拉刀磨削样件与检测结果

\section{3 设置优化分析}

为分析砂轮安装角 $\Sigma$ 与最大可行砂轮半径 $R_{\text {max }}$ 之间的关系并进行合理的选择, 离散的选择 5 个砂 轮安装角, 并按照不同的砂轮磨削越程量 $f_{2}$ 要求, 计算对应的砂轮最大可行半径。由此得砂轮安装角 $\Sigma$ 与最大砂轮可行半径 $R_{\text {max }}$ 的之间的关系如图 12 所示。从图 12 中可以清晰地观察到, 砂轮磨削越程 量的增大会直接导致最大可行砂轮半径的缩小; 随 着砂轮安装角的增大, 最大可行砂轮半径减小; 锐 角侧磨削最大可行砂轮半径远小于针角侧的最大可 行砂轮半径。

由此可见, 切削齿锐角侧的磨削是限制最大可
行砂轮半径的关键。为保证选用较大的砂轮半径, 一方面需要适当的控制较小的砂轮磨削越程量, 另 一方面, 需要选择较小的砂轮安装角 $\Sigma$ 。此外, 由 于砂轮安装角直接决定了砂轮磨削接触线的形态, 进而对切削齿的表面磨削质量产生影响, 一般建议 砂轮安装角 $\Sigma$ 适当小于切削齿的螺旋角 $\beta_{s}$ 即可。

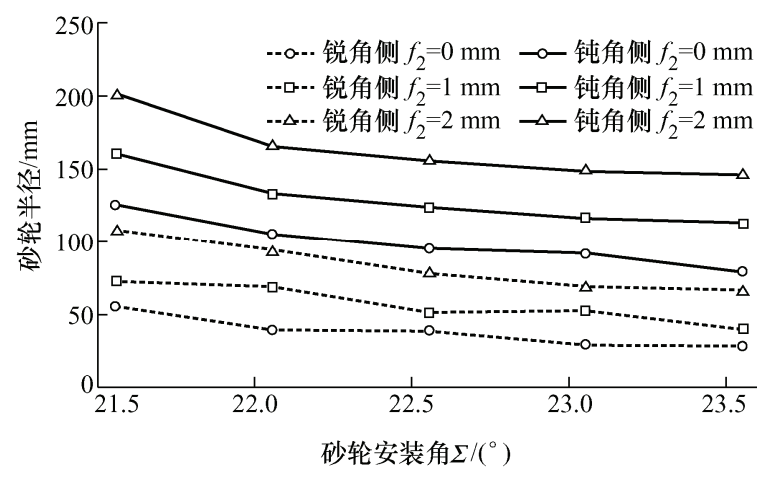

图 12 砂轮安装角与最大可行砂轮半径关系

\section{6 结论}

(1) 给出了螺旋内齿圈拉刀切削齿双螺旋结构 中的基本位移关系 $\left(u p_{x}, u p_{a}\right)$ 和 $\left(u g_{x}, u g_{a}\right)$, 并构建出面 向切削齿磨削定位的切削齿分布坐标参数化计算模 型。同时, 对精切齿段非对称台阶齿升量的拉削加 工原理进行了解析, 并给出了齿升量与齿序的参数 化编排方法, 为磨削路径的程序控制提供支持。

(2) 分析了精切齿磨削的基本工艺要素, 将螺旋拉 刀精切齿的磨削划分为三个阶段, 分别用于保证齿形 校准切削齿，形成锐角和针角侧切削齿的齿形磨削基 准，和保证基于跨棒距的齿形在机测量与控制实现。

(3) 提出了面向磨削效率优化的砂轮沿台阶型 齿升量从后向前轴向依次缩短磨削路径。同时, 一 方面指出优先进行钝角侧磨削, 以减小锐角侧修正 磨削的磨削路径长度, 另一方面规定每一齿升量的 磨削都以刀体周向为单位优先完成, 以确保周向切 削刃表面质量的一致性并便于砂轮修锐的程序控 制。最后, 给出了综合前述优化磨削工艺的磨削加 工路径控制算法, 确保了其工程应用性。

（4）基于切削齿的成型磨削接触线计算出发, 给出了精切齿侧面完整磨削以及砂轮与后一切削齿 无干涉的最大砂轮磨削行程的计算模型, 并进一步 构建出关于砂轮安装角的精切齿磨削可行砂轮半径 最大化的优化模型。实例分析指出切削齿锐角侧磨 削实现条件是限制砂轮尺寸的关键, 建议砂轮安装 角应略小于齿形螺旋角, 由此增加砂轮半径延长使 用寿命的同时可获取较好的磨削表面质量。 


\section{参 考 文 献}

[1] BOUZAKIS K D, LILI E, MICHAILIDIS N, et al. Manufacturing of cylindrical gears by generating cutting processes: A critical synthesis of analysis methods[J]. CIRP Annals-Manufacturing Technology, 2008, 57(2): 676-696.

[2] 李佳, 陈新春, 张鸿源. 圆柱齿轮别齿技术 $[\mathrm{J}]$. 机械工 程学报, 2011, 47(19): 193-198.

LI Jia, CHEN Xinchun, ZHANG Hongyuan. Slicing technology for cylindrical gears[J]. Journal of Mechanical Engineering, 2011, 47(19): 193-198.

[3] JIA K, ZHENG S, GUO J, et al. A surface envelopingassisted approach on cutting edge calculation and machining process simulation for skiving $[\mathrm{J}]$. The International Journal of Advanced Manufacturing Technology, 2019, $100(5-8):$ 1635-1645.

[4] SUTHERLAND J W, SALISURY E J, HOGE F W. A model for the cutting force system in the gear broaching process $[\mathrm{J}]$. International Journal of Machine Tools Manufacturing, 1997, 37(10): 1409-1421.

[5] SAVOY J, De SOUZA M M, DOMINGUES T G, et al. Technical and economic advantages of cold forged planetary-bevel gears developed with net shape teeth and splines[N]. SAE Technical Papers, 2013，36: 0551.

[6] GONÇALVES D A, SCHROETER R B. Modeling and simulation of the geometry and forces associated with the helical broaching process[J]. International Journal of Advanced Manufacturing Technology, 2016, 83(1-4): 205-215.

[7] JIA K, HONG J, ZHENG S, et al. An approach on wheel position and orientation calculation for helical broaching tool sharpening $[\mathrm{J}]$. International Journal of Advanced Manufacturing Technology, 2017, 92(5-8): 1991-2000.

[8] 贾康, 洪军, 张银行. 一种拉刀螺旋容屑槽前刀面磨削 砂轮安装位姿计算方法 $[\mathrm{J}]$. 机械工程学报, 2019, 55(11): 205-214.

JIA Kang, HONG Jun, ZHANG Yinhang. An approach on wheel setup calculation for helical rake flank sharpening of broaching tool $[\mathrm{J}]$. Journal of Mechanical Engineering, 2019, 55(11): 205-214.

[9] JIA K, HONG J, FAN L. Grinding path planning for the cutting teeth of helical broaching tool[J]. International Journal of Advanced Manufacturing Technology, 2016, 89(1): 1-14.

[10］袁哲俊, 刘华明. 金属切削刀具设计手册(单行本)拉刀 [M]. 北京：机械工业出版社，2009.

YUAN Z, LIU H. The design handbook of metal cutting tool (single) broach[M]. Beijing: China Machine Press, 2009.

作者简介: 贾康, 男, 1987 年出生, 博士, 助理研究员。主要研究方向 为数字化制造、大尺寸空间测量。

E-mail: jiakang@xjtu.edu.cn

洪军(通信作者), 男, 1968 年出生, 博士, 教授, 博士研究生导师。主 要研究方向为数字化设计、数字化装配技术以及高速精密主轴系统。

E-mail: jhong@mail.xjtu.edu.cn 